\title{
A NOTE ON QUADRATIC JORDAN ALGEBRAS OF DEGREE $3\left(^{1}\right)$
}

\author{
BY \\ M. L. RACINE
}

\begin{abstract}
McCrimmon has defined a class of quadratic Jordan algebras of degree 3 obtained from a cubic form, a quadratic mapping and a base point. The structure of such an algebra containing no absolute zero-divisor is determined directly. A simple proof of Springer's Theorem on isomorphism of reduced simple exceptional quadratic Jordan algebras is given.
\end{abstract}

1. Introduction and basic concepts. A unital quadratic Jordan algebra over a field $\Phi$ is a triple $(\mathscr{J}, U, 1)$ where $\mathscr{J}$ is a $\Phi$ vector space, 1 a distinguished element of $\mathscr{J}$, and $U$ is a mapping $a \rightarrow U_{a}$ of $\mathscr{J}$ into $\operatorname{End}_{\Phi}(\mathscr{J})$ satisfying the following axioms:

QJ1. $U$ is $\Phi$-quadratic, that is $U_{\lambda a}=\lambda^{2} U_{a}, \lambda \in \Phi, a \in \mathscr{J}$, and $U_{a, b}=U_{a+b}-U_{a}-U_{b}$ is $\Phi$-bilinear in $a$ and $b$.

QJ2. $U_{1}=1$.

QJ3. $U_{a U_{b}}=U_{b} U_{a} U_{b}$.

QJ4. If $V_{a, b}$ is defined by $x V_{a, b}=a U_{x, b}$, then $U_{b} V_{a, b}=V_{b, a} U_{b}$.

QJ5. QJ(1)-(4) hold for $\mathscr{J}^{\mathrm{P}}=\mathscr{J} \otimes_{\Phi} \mathrm{P}, \mathrm{P}$ any field extension of $\Phi$.

Powers are defined inductively: $x^{0}=1, x^{1}=x$, and $x^{n+2}=x^{n} U_{x}$. An element $z \in \mathscr{J}, z \neq 0$, is said to be an absolute zero divisor if $U_{z}=0$. An element $a \in \mathscr{J}$ is invertible if there exists a $b \in \mathscr{J}$ with $b U_{a}=a$ and $b^{2} U_{a}=1$; such a $b$ is uniquely determined and is denoted $a^{-1}$. We say that $\mathscr{J}$ is a quadratic Jordan division algebra if every nonzero element of $\mathscr{J}$ is invertible.

Let $\mathscr{J}$ be a $\Phi$ vector space. Assume given a quadratic form $Q$ on $\mathscr{J}$ and a distinguished element $1 \in \mathscr{J}$ with $Q(1)=1$. Define $a U_{b}=Q\left(a, b^{*}\right) a-Q(a) b^{*}$ where $b^{*}=Q(b, 1) 1-b$ and $Q(a, b)=Q(a+b)-Q(a)-Q(b)$. Jacobson and McCrimmon have shown in [5] that this defines a quadratic Jordan algebra, the quadratic Jordan algebra of the quadratic form $Q$ with base point 1 , denoted $\mathscr{J}(Q, 1)$.

Let $\mathscr{J}$ be a $\Phi$ vector space. Assume given (i) a cubic form $N$ on $\mathscr{J}$ with values in $\Phi$ (so $N$ is homogeneous of degree 3 and $N(x+y)=N(x)+\Delta_{x}^{y} N+\Delta_{y}^{x} N+N(y)$, where $\Delta_{x}^{y} N$ is the directional derivative of $N$ in the direction $y$, evaluated at $x$ ),

Received by the editors January 20, 1971.

AMS 1970 subject classifications. Primary 17C10, 17C20, 17C40; Secondary 10C10.

Key words and phrases. Quadratic Jordan algebras, cubic form, isomorphism, exceptional Jordan algebra.

(1) This research was partially supported by the National Science Foundation (NSF-GP9572).

Copyright $(1)$ 1972, American Mathematical Society 
(ii) a quadratic mapping $x \rightarrow x^{\#}$ in $\mathscr{J}$, and (iii) a distinguished element $1 \in \mathscr{J}$, related by

(1) $x^{\# \#}=N(x) x$.

(2) $N(1)=1$.

(3) $T\left(x^{\#}, y\right)=\Delta_{x}^{y} N$, where $T(x, y)=-\Delta_{1}^{x} \Delta^{y} \log N$.

(4) $1^{\#}=1$.

(5) $1 \times y=T(y) 1-y$, where $T(y)=T(y, 1)$ and $x \times y=(x+y)^{\#}-x^{\#}-y^{\#}$.

Assume moreover that these hold for any $\mathscr{J}^{\mathrm{P}}, \mathrm{P}$ any field extension of $\Phi$. Introduce a $U$ operator.

(6) $y U_{x}=T(x, y) x-x^{\#} \times y$.

McCrimmon has shown in [6] that $(\mathscr{J}, U, 1)$ is a quadratic Jordan algebra which we denote $\mathscr{J}(N, \#, 1)$.

We recall also that a composition algebra $\mathscr{C}$ over $\Phi$ is a unital $\Phi$-algebra (not necessarily associative; unital = contains a unit element 1 ) with a nondegenerate quadratic form $n$ of $\mathscr{C}$ into $\Phi$ such that $n(1)=1$ and $n(a b)=n(a) n(b)$. We refer to [1] for the determination of composition algebras and their properties. Let $\mathscr{C}$ be a composition algebra over $\Phi, \mathscr{C}_{3}$ the algebra of $3 \times 3$ matrices over $\mathscr{C}, \gamma$ the diagonal matrix diag $\left\{\gamma_{1}, \gamma_{2}, \gamma_{3}\right\}$ where the $\gamma_{i} \neq 0$ are in $\Phi$. Then $J_{\gamma}: x \rightarrow \gamma^{-1} \bar{x}^{t} \gamma$ is an involution in $\mathscr{C}_{3}$ if $\bar{x}=\left(\bar{x}_{i j}\right)$ for $x=\left(x_{i j}\right)$ and $x^{t}$ is the transpose of $x$. Let $\mathscr{H}\left(\mathscr{C}_{3}, J_{\gamma}\right)$ be the $\Phi$-space of matrices satisfying $x^{J_{y}}=x$ and whose diagonal entries lie in $\Phi$. McCrimmon has shown in [6] that if one defines

$$
\begin{aligned}
N(x) & =\alpha_{1} \alpha_{2} \alpha_{3}-\alpha_{1} \gamma_{3}^{-1} \gamma_{2} n\left(a_{1}\right)-\alpha_{2} \gamma_{1}^{-1} \gamma_{3} n\left(a_{2}\right)-\alpha_{3} \gamma_{2}^{-1} \gamma_{1} n\left(a_{3}\right)-t\left(a_{1} a_{2} a_{3}\right), \\
x^{\#} & =\left(\begin{array}{lll}
\alpha_{2} \alpha_{3}-\gamma_{3}^{-1} \gamma_{2} n\left(a_{1}\right) & \gamma_{1}^{-1} \gamma_{2} \overline{a_{1} a_{2}}-\alpha_{3} a_{3} & a_{3} a_{1}-\gamma_{1}^{-1} \gamma_{3} \alpha_{2} \bar{a}_{2} \\
a_{1} a_{2}-\gamma_{2}^{-1} \gamma_{1} \alpha_{3} \bar{a}_{3} & \alpha_{3} \alpha_{1}-\gamma_{1}^{-1} \gamma_{3} n\left(a_{2}\right) & \gamma_{2}^{-1} \gamma_{3} \bar{a}_{2} a_{3}-\alpha_{1} a_{1} \\
\gamma_{3}^{-1} \gamma_{1} \bar{a}_{3} a_{1}-\alpha_{2} a_{2} & a_{2} a_{3}-\gamma_{3}^{-1} \gamma_{2} \alpha_{1} \bar{a}_{1} & \alpha_{1} \alpha_{2}-\gamma_{2}^{-1} \gamma_{1} n\left(a_{3}\right)
\end{array}\right)
\end{aligned}
$$

for

$$
x=\left(\begin{array}{ccc}
\alpha_{1} & a_{3} & \gamma_{1}^{-1} \gamma_{3} \bar{a}_{2} \\
\gamma_{2}^{-1} \gamma_{1} \bar{a}_{3} & \alpha_{2} & a_{1} \\
a_{2} & \gamma_{3}^{-1} \gamma_{2} \bar{a}_{1} & \alpha_{3}
\end{array}\right),
$$

then the cubic form $N$ on $\mathscr{J}=\mathscr{H}\left(\mathscr{C}_{3}, J_{y}\right), 1$ the unit matrix and $x^{\#}$ satisfy (1)-(5) in $\mathscr{J}^{\mathrm{P}}$ for every P. Hence if $U$ is defined by $(6)$ then $\mathscr{J}(N, \#, 1)$ is a quadratic Jordan algebra. From now on $\mathscr{H}\left(\mathscr{C}_{3}, J_{\gamma}\right)$ will denote the space $\mathscr{H}\left(\mathscr{C}_{3}, J_{y}\right)$ with this quadratic Jordan structure.

The following theorem has been proved by Springer [8] when the characteristic of $\Phi \neq 2$ or 3 .

THEOREM 1. Let $\mathscr{J}=\mathscr{J}(N, \#, 1)$ be a unital quadratic Jordan algebra over a field $\Phi$. If $\mathscr{J}$ has no absolute zero divisor then $\mathscr{J}$ is either (i) a quadratic Jordan division algebra, (ii) a direct sum $\Phi \oplus \mathscr{J}\left(Q, 1^{\prime}\right)$, where $\mathscr{J}\left(Q, 1^{\prime}\right)$ is the quadratic Jordan algebra of the quadratic form $Q$ with base point $1^{\prime}$, or (iii) $\mathscr{H}\left(\mathscr{C}_{3}, J_{y}\right)$. 
This result follows also from structure theory [4]. Our purpose is to give a direct proof of this theorem which depends only on the properties of $N$ and \# obtained in [6] and on those of $\mathscr{J}(Q, 1)$ found in [5]. We also prove Springer's Theorem on isomorphism of $\mathscr{H}\left(\mathscr{C}_{3}, J_{\gamma}\right)$ 's. This result is new in characteristic 2.

The author would like to express his gratitude to Professor Jacobson for his valuable suggestions. Thanks are also due to Professor McCrimmon for his helpful comments.

2. The proof of Theorem 1. Let $\mathscr{J}$ be any $\Phi$ vector space equipped with a cubic norm $N$, a mapping \# and an element 1 satisfying (1)-(5), so we have the quadratic Jordan algebra $\mathscr{J}(N, \#, 1)$. The following identities can be found in [6]. Let $x, y, z \in \mathscr{J}$.

(9) $T(1)=3$.

(10) $x^{\#} \times y^{\#}+(x \times y)^{\#}=T\left(x^{\#}, y\right) y+T\left(y^{\#}, x\right) x$.

(11) $x^{\#} \times(y \times z)+(x \times y) \times(x \times z)=T\left(x^{\#}, y\right) z+T\left(x^{\#}, z\right) y+T(y \times z, x) x$.

(12) $T(x \times y, z)=T(x, y \times z)$.

(13) $T(x \times y)=T(x) T(y)-T(x, y)$.

(14) $x^{\#} \times x=\left[T\left(x^{\#}\right) T(x)-N(x)\right] 1-T\left(x^{\#}\right) x-T(x) x^{\#}$.

(15) $x^{3}-T(x) x^{2}+S(x) x-N(x) 1=0$, where

(16) $S(x)=T\left(x^{\#)}\right.$.

(17) $x^{\#}=x^{2}-T(x) x+S(x) 1$.

(18) $\left(y U_{x}\right)^{\#}=y^{\#} U_{x}$.

Also an element $x \in \mathscr{J}$ is invertible if and only if $N(x) \neq 0$, in which case

(19) $x^{-1}=N(x)^{-1} x^{\#}$.

(20) $T\left(x U_{y}, z\right)=T\left(x, z U_{y}\right)$ follows immediately from (12) and (6).

From (5) $x=-x \times 1+T(x) 1$, so $x \times(x \times y)=-(x \times 1) \times(x \times y)+T(x) 1 \times(x \times y)$ $=x^{\#} \times(1 \times y)-T\left(x^{\#}, y\right) 1-T\left(x^{\#}, 1\right) y-T(1 \times y, x) x+T(x)[T(x \times y) 1-x \times y]$ $=x^{\#} \times(T(y) 1-y)-T\left(x^{\#}, \quad y\right) 1-T\left(x^{\#}\right) y-T(1, \quad x \times y) x+T(x) T(x \times y) 1-T(x) x \times y$ $=T(y) T\left(x^{\#}\right) 1-T(y) x^{\#}-x^{\#} \times y-T\left(x^{\#}, y\right) 1-T\left(x^{\#}\right) y-T(x \times y) x+T(x) T(x \times y) 1$ $-T(x) x \times y=\left[T\left(x^{\#} \times y\right)+T(x) T(x \times y)\right] 1-T(y) x^{\#}-x^{\#} \times y-T\left(x^{\#}\right) y-T(x \times y) x$ $-T(x) x \times y$ by (11), (12), (13) and (5) and we have

$$
\begin{aligned}
x \times(x \times y)= & {\left[T\left(x^{\#} \times y\right)+T(x) T(x \times y)\right] 1 } \\
& -T(y) x^{\#}-x^{\#} \times y-T\left(x^{\#}\right) y-T(x \times y) x-T(x) x \times y .
\end{aligned}
$$

An idempotent $e \neq 0$ is said to be primitive if $e^{\#}=0$. (This implies $T(e)=1$, and the converse holds if the characteristic of $\Phi \neq 2$.)

LEMmA 1. If $\mathscr{J}=\mathscr{J}(N, \#, 1)$ does not contain any absolute zero divisors and is not a quadratic Jordan division algebra then $\mathscr{J}$ contains a primitive idempotent.

Proof. If $\mathscr{J}$ is not a quadratic Jordan division algebra, $N(x)=0$ for some $x \neq 0$, so $x^{\# \#}=0$ and there is a $y \neq 0$ (either $y=x^{\#}$ if $x^{\#} \neq 0$, or $y=x$ if $x^{\#}=0$ ) with $y^{\#}=0$. Then $y^{2}=T(y) y$; if $T(y) \neq 0$ then $e=T(y)^{-1} y$ is an idempotent and $e^{\#}=0$, while if 
$T(y)=0, y^{2}=0$. Suppose $T(y)=0$. Since $y$ is not an absolute zero divisor and ${ }_{z} U_{y}=T(y, z) y-y^{\#} \times z=T(y, z) y$, there is a $z$ such that $T(y, z)=1$. Let $a=y \times z$, $T(a)=T(y \times z)=T(y) T(z)-T(y, z)=-1 \quad$ and $\quad a \neq 0 ; \quad a^{\#}=(y \times z)^{\#}=T\left(y^{\#}, z\right) z+$ $T\left(z^{\#}, y\right) y-y^{\#} \times z^{\#}=T\left(z^{\#}, y\right) y$, hence $T\left(a^{\#}\right)=0=N(a)$. Let $e=a^{\#}-a ; T(e)=1$ and $e^{\#}$ $=\left(a^{\#}-a\right)^{\#}=a^{\# \#}-a^{\#} \times a+a^{\#}=N(a) a-\left[T\left(a^{\#}\right) T(a)-N(a)\right] 1+T\left(a^{\#}\right) a+T(a) a^{\#}+a^{\#}$ $=-a^{\#}+a^{\#}=0$. Hence $0=e^{\#}=e^{2}-T(e) e+T\left(e^{\#}\right) 1=e^{2}-e$ and $e$ is a primitive idempotent.

Assume from now on that $\mathscr{J}$ does not contain absolute zero divisors and is not a quadratic Jordan division algebra. Therefore $\mathscr{J}$ contains a primitive idempotent $e$. Let $f=1-e ; 1-e=T(e) 1-e=1 \times e=(e+f) \times e=e \times e+f \times e=2 e^{\#}+f \times e=f \times e$ and $1=1^{\#}=(e+f)^{\#}=e^{\#}+e \times f+f^{\#}=e \times f+f^{\#}$, so

(22) $e \times f=f, f^{\#}=e$.

By (12) $T(f, e)=T(f \times e, e)=T(f, e \times e)=T\left(f, 2 e^{\sharp}\right)=0$, so we have

(23) $T(e, f)=0$.

Note that $T(f)=T(1-e)=T(1)-T(e)=2$ and $e=f^{\#}=f^{2}-T(f) f+T\left(f^{\#}\right) 1=f^{2}-2 f$ $+1=f^{2}-f+e$, so $f^{2}=f$ and $f$ is an idempotent. Therefore $\left(U_{f}\right)^{2}=U_{f} U_{1} U_{f}=U_{1 U_{f}}$ $=U_{f^{2}}=U_{f}$; similarly $\left(U_{e}\right)^{2}=U_{e}$. Moreover $x U_{e} U_{f}=T(e, x) e U_{f}=T(e, x)[T(f, e) f$ $\left.-f^{\#} \times e\right]=-T(e, x) e \times e=0 \quad$ and $\quad x U_{f} U_{e}=T(f, x) f U_{e}-(e \times x) U_{e}=T(f, x) T(e, f) e$ $-T(e \times x, e) e=-T(x, e \times e) e=0$, so $U_{e} U_{f}=0=U_{f} U_{e}$. Therefore $U_{e}, U_{f}$ and $U_{e, f}=$ $U_{1}-U_{e}-U_{f}$ are orthogonal projections; hence

(24) $\mathscr{J}=\mathscr{J}_{1} \oplus \mathscr{J}_{1 / 2} \oplus \mathscr{J}_{0}$ where $\mathscr{J}_{1}=\mathscr{J} U_{e}, \mathscr{J}_{1 / 2}=\mathscr{J} U_{e, f}$ and $\mathscr{J}_{0}=\mathscr{J} U_{f}$.

This is the Peirce decomposition of $\mathscr{J}$ with respect to $e$ and $\mathscr{J}_{i}$ will be denoted $\mathscr{F}_{i}(e)$, if needed, to emphasize that the decomposition is taken with respect to $e$. By (20) we have

(25) $T\left(\mathscr{J}_{i}, \mathscr{J}_{j}\right)=0$ if $i \neq j, i, j=0, \frac{1}{2}, 1$.

In particular $e=1 U_{e} \in \mathscr{J}_{1}$ and $f=1 U_{f} \in \mathscr{J}_{0}$, so

(26) $T\left(\mathscr{J}_{1 / 2}, e\right)=0, T\left(\mathscr{J}_{1 / 2}, f\right)=0$ and $T\left(\mathscr{J}_{1 / 2}\right)=T\left(\mathscr{J}_{1 / 2}, 1\right)=0$.

Note that

$$
\begin{aligned}
x U_{e} & =T(x, e) e, \\
x U_{f} & =T(x, f) f-e \times x, \\
x U_{e, f} & =T(x, e) f+T(x, f) e-f \times x .
\end{aligned}
$$

Let $x \in \mathscr{J}_{1 / 2}$, then (26) and (27) imply $x=-f \times x$, and $1 \times x=T(x) 1-x=-x$ implies $e \times x=0$. Conversely if $x \in \mathscr{J}$ with $T(x)=0$ and $e \times x=0$ then $x=-f \times x$ and $0=T(x)=-T(f \times x)=T(f, x)-T(x) T(f)=T(f, x)$ so $T(e, x)=0$ and by $(27) x \in \mathscr{J}_{1 / 2}$.

(28) $x \in \mathscr{J}_{1 / 2}(e)$ if and only if $T(x)=0$ and $e \times x=0$.

If $y \in \mathscr{J}$ with $T(y)=0$ and $e \times y=-y$ then $0=T(y)=-T(e \times y)=T(e, y)$ $-T(e) T(y)=T(e, y)$ so $T(f, y)=0$ and by $(27) y \in \mathscr{L}_{0}(e)$.

(29) $T(y)=0$ and $e \times y=-y$ imply $y \in \mathscr{J}_{0}(e)$.

Let $x \in \mathscr{J}_{1 / 2}$, then $x=-f \times x$ and $x^{\#}=(-f \times x)^{\#}=T\left(f^{\#}, x\right) x+T\left(x^{\#}, f\right) f-f^{\#} \times x^{\#}$ $=T\left(x^{\#}, f\right) f-e \times x^{\#} \in \mathscr{J}_{0}$ by (10), (22), (26) and (27), so

(30) $x^{\#} \in \mathscr{J}_{0}$ for $x \in \mathscr{J}_{1 / 2}$. 
If $x \in \mathscr{J}_{0}$ then $x^{\#}=\left(x U_{f}\right)^{\#}=x^{\#} U_{f \#}=x^{\#} U_{e}=T\left(x^{\#}, e\right) e$ and $S(x)=T\left(x^{\#}\right)=T\left(x^{\#}, e\right) T(e)$ $=T\left(x^{\#}, e\right)$, so we have

(31) $x^{\#}=S(x) e \in \mathscr{J}_{1}$ for $x \in \mathscr{J}_{0}$.

LEMma 2 (FAULKNER [2]). $\mathscr{J}_{0}=\mathscr{J}(S, f)$, the quadratic Jordan algebra of the quadratic form $S$ restricted to $\mathscr{J}_{0}$ with base point $f$.

Proof. Let $x, y \in \mathscr{J}_{0}$, then $x=T(x, f) f-e \times x$. Define $x^{*}=e \times x=T(x, f) f-x$. We have $y U_{x}=T(x, y) x-x^{\#} \times y=T(x, y) x-S(x) e \times y=T(x, y) x-S(x) y^{*}$. But $S\left(x, y^{*}\right)=T\left(x \times y^{*}\right)=T(x) T\left(y^{*}\right)-T\left(x, y^{*}\right)=T(x) T(e \times y)-T(x, e \times y)=T(x) T(y, e \times 1)$ $-T(x \times y, e)=T(x) T(y, f)-T(x \times y)=T(x) T(y)-T(x \times y)=T(x, y)$ by (12), (13), (25) and (31). Hence $y U_{x}=S\left(x, y^{*}\right) x-S(x) y^{*}$. Finally $S(f)=T\left(f^{\#}\right)=T(e)=1$ and $f^{*}=e \times f=f$, so that $x^{*}=T(x, f) f-x=S\left(x, f^{*}\right) f-x=S(x, f) f-x$.

We shall now show that if $\mathscr{J}_{1 / 2}=\{0\}$ then we have case (ii), that is $\mathscr{J}=\mathscr{J}_{1} \oplus \mathscr{J}_{0}$ is an algebra direct sum. Let $x, y \in \mathscr{J}_{0} ; x U_{e}=T(e, x) e=0$ by $(26), e U_{x}=T(x, e) x$ $-x^{\#} \times e=-S(x) e \times e=0, e U_{e, x}=T(e, e) x+T(e, x) e-(e \times x) \times e=x-e \times(T(x) f-x)$ $=x-T(x) f+e \times x=0$ by (27) and (22). Finally $y U_{e, x}=T(y, e) x+T(y, x) e-(e \times x) \times y$ $=T(y, x) e-(T(x) f-x) \times y=T(x, y) e-T(x)(1 \times y-e \times y)-T(x \times y) e=T(x, y) e-$ $T(x)(T(y) 1-y-T(y) f+y)-T(x \times y) e=0$ by (13), (27) and (31). Hence if $x, x^{\prime} \in \mathscr{J}_{1}$ and $y, y^{\prime} \in \mathscr{J}_{0}$ then $(x+y) U_{x^{\prime}+y^{\prime}}=x U_{x^{\prime}}+y U_{y^{\prime}}$ so we have the algebra direct decomposition $\mathscr{J}=\mathscr{J}_{0} \oplus \mathscr{J}_{1}$.

Assume we are not in case (ii), so $\mathscr{J}_{1 / 2} \neq\{0\}$. Suppose $\left(\mathscr{J}_{1 / 2}\right)^{\#}=\{0\}$. Since $x \in \mathscr{J}_{1 / 2}$, $x \neq 0$, is not an absolute zero divisor, there exists a $y$, which by (25) may be taken in $\mathscr{J}_{1 / 2}$, with $T(x, y) \neq 0$. But, by $(26), T(x)=0$ and $x \times y=(x+y)^{\#}-x^{\#}-y^{\#}=0$ which yields $T(x, y)=T(x) T(y)-T(x \times y)=0$, a contradiction. Therefore $\mathscr{J}_{1 / 2} \neq\{0\}$

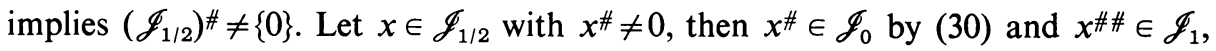
by (31). However $x^{\# \#}=N(x) x \in \mathscr{J}_{1 / 2}$. Therefore $x^{\# \#}=0$ and $N(x)=0$. Since $e^{\#}=0$, (27) and (31) imply

$$
N(x)=0 \text { for } x \in \mathscr{J}_{i}, \quad i=0, \frac{1}{2}, 1 .
$$

Also if $x \in \mathscr{J}_{1 / 2}$ then $N(x)=0$ implies that $S\left(x^{\#)}\right)=T\left(x^{\# \#}\right)=T(N(x) x)=0$. Since we can choose $x \in \mathscr{J}_{1 / 2}$ so that $x^{\#} \neq 0$ and $x^{\#} \in \mathscr{J}_{0}$ it is clear that the restriction of $S$ to $\mathscr{J}_{0}$ is isotropic. Let $y \in \mathscr{J}_{0}, y \neq 0$. If $S(y)=0$ then $y^{\#}=0$ by (31) and $y$ not an absolute zero divisor implies $T(y, z) \neq 0$ for some $z \in \mathscr{J}_{0}$. In the proof of Lemma 2 $T(y, z)$ was seen to equal $S\left(y, z^{*}\right)$, therefore the restriction of $S$ to $\mathscr{J}_{0}$ is nondegenerate. From Theorem 11 of [5] we know that $\mathscr{J}_{0}$ contains a primitive idempotent unless characteristic $\Phi=2$ and $T\left(\mathscr{J}_{0}\right)=0$. In that case consider $e^{\prime}=e+x+x^{\#}$, $x \in \mathscr{J}_{1 / 2}, x^{\#} \neq 0 ; T\left(e^{\prime}\right)=T(e)=1$. We have $\left(e^{\prime}\right)^{\#}=e^{\#}+x^{\#}+x^{\# \#}+e \times x+e \times x^{\#}+x \times x^{\#}$ $=0$ since $e^{\#}=0=x^{\# \#}, e \times x=0$ by $(28), e \times x^{\#}=T\left(x^{\#}\right) f-x^{\#}=-x^{\#}$ since $T\left(\mathscr{J}_{0}\right)$ $=0$, and $x \times x^{\#}=\left[T\left(x^{\#}\right) T(x)-N(x)\right] 1-T\left(x^{\#}\right) x-T(x) x^{\#}=0$ by (26) and (32). So $e^{\prime}$ is a primitive idempotent and $T\left(\mathscr{J}_{0}\left(e^{\prime}\right)\right)=T\left(\mathscr{J} U_{f^{\prime}}\right)=T\left(\mathscr{J}, f^{\prime}\right)=T\left(\mathscr{J}, f-x-x^{\sharp}\right)=$ $-T\left(\mathscr{J}, x^{\#}\right)$ since $T(x, \mathscr{J})=T\left(x, \mathscr{J}_{1 / 2}\right)=T\left(x \times \mathscr{J}_{1 / 2}\right) \subset T\left(\mathscr{J}_{0}\right)=0$. Since $x^{\#}$ is not an absolute zero divisor, $x^{\# \#}=0$ implies $T\left(\mathscr{J}, x^{\#}\right) \neq 0$. Accordingly we may assume the 
existence of a primitive idempotent $e_{1}$ such that $\mathscr{J}_{0}\left(e_{1}\right)$ contains a primitive idempotent $e_{2}$. Two primitive idempotents $e_{1}$ and $e_{2}$ are said to be orthogonal $\left(e_{1} \perp e_{2}\right)$ if $e_{2} \in \mathscr{J}_{0}\left(e_{1}\right)$. The definition is symmetric, that is $e_{2} \in \mathscr{J}_{0}\left(e_{1}\right)$ implies $e_{1} \in \mathscr{J}_{0}\left(e_{2}\right)$. Indeed $e_{2}=T\left(e_{2}, 1-e_{1}\right)\left(1-e_{1}\right)-e_{1} \times e_{2}$ by $(27)=T\left(e_{2}\right)\left(1-e_{1}\right)-e_{1} \times e_{2}=1-e_{1}-e_{1} \times e_{2}$ and so $e_{1}=1-e_{2}-e_{1} \times e_{2}=T\left(e_{1}, 1-e_{2}\right)\left(1-e_{2}\right)-e_{2} \times e_{1}=e_{1} U_{1-e_{2}} \in \mathscr{J}_{0}\left(e_{2}\right)$. Let $e_{3}=1-e_{1}$ $-e_{2}=e_{1} \times e_{2} ; e_{3}$ is a primitive idempotent by (9) and (10). We have $e_{1} \perp e_{2}$, $e_{1} \perp e_{3}$ and $e_{2} \perp e_{3}$ since the definition is symmetric. By (21), $e_{1} \times e_{3}=e_{2}$ and $e_{2} \times e_{3}=e_{1}$. Assume from now on that $i, j, k \in\{1,2,3\}$ and are distinct. Define $\mathscr{J}_{i i}=\mathscr{J}_{1}\left(e_{i}\right), \mathscr{J}_{i j}=\mathscr{J} U_{e_{i}, e_{j}}$. Now $x U_{e_{i}, e_{j}} U_{e_{i}, e_{k}}=\left[T\left(x, e_{i}\right) e_{j}+T\left(x, e_{j}\right) e_{i}-e_{k} \times x\right] U_{e_{i}, e_{k}}$ $=T\left(x, e_{i}\right)\left[T\left(e_{j}, e_{i}\right) e_{k}+T\left(e_{j}, e_{k}\right) e_{i}-e_{j} \times e_{j}\right]+T\left(x, e_{j}\right)\left[T\left(e_{i}, e_{i}\right) e_{k}+T\left(e_{i}, e_{k}\right) e_{i}-e_{j} \times e_{i}\right]-$ $T\left(e_{k} \times x, e_{i}\right) e_{k}-T\left(e_{k} \times x, e_{k}\right) e_{i}+\left(e_{k} \times e_{i}\right) \times\left(e_{k} \times x\right)=-T\left(e_{k} \times x, e_{i}\right) e_{k}-e_{k}^{\#} \times\left(e_{i} \times x\right)+$ $T\left(e_{k}^{\#}, e_{i}\right) x+T\left(e_{k}^{\#}, x\right) e_{i}+T\left(e_{i} \times x, e_{k}\right) e_{k}=0$ by (11), (12) and (25), so $U_{e_{i}, e_{j}}$ and $U_{e_{i}, e_{k}}$ are orthogonal operators. Moreover $x U_{e_{i}, e_{j}}^{2}=\left[T\left(x, e_{i}\right) e_{j}+T\left(x, e_{j}\right) e_{i}-e_{k} \times x\right] U_{e_{i}, e_{j}}$ $=T\left(x, e_{i}\right)\left[T\left(e_{j}, e_{i}\right) e_{j}+T\left(e_{j}, e_{j}\right) e_{i}-e_{k} \times e_{j}\right]+T\left(x, e_{j}\right)\left[T\left(e_{i}, e_{i}\right) e_{j}+T\left(e_{i}, e_{j}\right) e_{i}-e_{k} \times e_{i}\right]-$ $T\left(x \times e_{k}, e_{i}\right) e_{j}-T\left(x \times e_{k}, e_{j}\right) e_{i}+e_{k} \times\left(e_{k} \times x\right)=-T\left(x, e_{j}\right) e_{j}-T\left(x, e_{i}\right) e_{i}+\left[T\left(e_{k}^{\#} \times x\right)+\right.$ $\left.T\left(e_{k}\right) T\left(e_{k} \times x\right)\right] 1-T(x) e_{k}^{\#}-e_{k}^{\#} \times x-T\left(e_{k}^{\#}\right) x-T\left(e_{k} \times x\right) e_{k}-T\left(e_{k}\right) e_{k} \times x=-T\left(x, e_{j}\right) e_{j}-$ $T\left(x, e_{i}\right) e_{i}+T\left(x, e_{k} \times 1\right) 1-T\left(x, e_{k} \times 1\right) e_{k}-e_{k} \times x=T\left(x, e_{i}\right) e_{j}+T\left(x, e_{j}\right) e_{i}-e_{k} \times x=x U_{e_{i}, e_{j}}$ by (5), (12), (21) and (25), and we have $U_{e_{i}, e_{j}}^{2}=U_{e_{i}, e_{j}}$. Also $U_{e_{l}} \perp U_{e_{i}, e_{j}+e_{k}}=U_{e_{i}, e_{j}}$ $+U_{e_{i}, e_{k}}$, so $0=U_{e_{i}}\left(U_{e_{i}, e_{j}}+U_{e_{i}, e_{k}}\right) U_{e_{i}, e_{j}}=U_{e_{i}} U_{e_{i}, e_{j}}$ and similarly $U_{e_{i}, e_{j}} U_{e_{i}}=0$. Hence $U_{e_{i}} \perp U_{e_{i}, e_{j}}$. Then $\mathscr{J}=\mathscr{J} U_{e_{i}} \oplus \mathscr{J} U_{e_{i}, e_{j}+e_{k}} \oplus \mathscr{J} U_{e_{j}+e_{k}}=\mathscr{J} U_{e_{i}} \oplus\left(\mathscr{J} U_{e_{i}, e_{j}} \oplus \mathscr{J} U_{e_{i}, e_{k}}\right)$ $\oplus\left(\mathscr{J} U_{e_{j}} \oplus \mathscr{J} U_{e_{j}, e_{k}} \oplus \mathscr{J} U_{e_{k}}\right)$. Combining this with the previous results on the Peirce decomposition of $\mathscr{J}$ with respect to a primitive idempotent $e$ we obtain

$$
\begin{aligned}
& \mathscr{J}=\oplus \sum_{i=1}^{3} \mathscr{J}_{i i} \oplus \sum_{i<j} \mathscr{J}_{i j}, \quad \mathscr{J}_{i j}=\mathscr{J}_{1 / 2}\left(e_{i}\right) \cap \mathscr{J}_{1 / 2}\left(e_{j}\right), \quad \mathscr{J}_{i i}=\Phi e_{i} . \\
& T\left(\mathscr{J}_{i j}, \mathscr{J}_{i k}\right)=0 . \\
& e_{i} \times e_{j}=e_{k} . \\
& e_{i} \times x=-x, \quad e_{j} \times x=0=e_{k} \times x \quad \text { for } x \in \mathscr{J}_{j k} . \\
& x^{\#}=S(x) e_{i} \text { for } x \in \mathscr{J}_{j k} .
\end{aligned}
$$

Let $a \in \mathscr{J}_{i j}, b \in \mathscr{J}_{j k}$, then $a, b \in \mathscr{J}_{1 / 2}\left(e_{j}\right)$, so $a \times b \in \mathscr{J}_{0}\left(e_{j}\right)$. But $T\left(a \times b, e_{i}\right)=T\left(b, a \times e_{i}\right)$ $=T(b, 0)=0$ and $T\left(a \times b, e_{k}\right)=T\left(a, b \times e_{k}\right)=T(a, 0)=0$ by (33) and we have

(34) $a \times b \in \mathscr{J}_{i k}$ for $a \in \mathscr{J}_{i j}, b \in \mathscr{J}_{j k}$.

If $\mathscr{J}_{i j}=\{0\}=\mathscr{J}_{j k}$ then $\mathscr{J}_{1 / 2}\left(e_{j}\right)=0$ and we have case (ii). Assume that only one, say, $\mathscr{J}_{i k}=\{0\}$, and $\mathscr{J}_{i j} \neq\{0\}, \mathscr{J}_{j k} \neq\{0\}$. Arguing as above we may pick $a \in \mathscr{J}_{1 / 2}\left(e_{i}\right)$ $=\mathscr{J}_{i j}, \quad b \in \mathscr{J}_{1 / 2}\left(e_{k}\right)=\mathscr{J}_{j k}$ with $a^{\#}=S(a) e_{k} \neq 0, b^{\#}=S(b) e_{i} \neq 0 ; a \times b \in \mathscr{J}_{i k}$ and so $a \times b=0$. But $(a+b)^{\#}=a^{\#}+a \times b+b^{\#}=S(a) e_{k}+S(b) e_{i}$ and $(a+b)^{\# \#}=S(a) S(b) e_{i} \times e_{k}$ $=S(a) S(b) e_{j} \neq 0$ contradicting (32). Therefore if $\mathscr{J}$ is not of the form (i) or (ii), $\mathscr{J}_{12}, \mathscr{J}_{23}, \mathscr{J}_{31}$ are nonzero and by the argument on p. 97 each contains an element whose \# is nonzero. Let $a \in \mathscr{J}_{i j}, b \in \mathscr{J}_{i k}$. By $(10)(a \times b)^{\#}=T\left(a^{\#}, b\right) b+T\left(b^{\#}, a\right) a$ $-a^{\#} \times b^{\#}=-S(a) S(b) e_{k} \times e_{j}=-S(a) S(b) e_{i}$, so $S(a \times b)=T\left((a \times b)^{\#}\right)=-S(a) S(b)$ and we have

(35) $S(a \times b)=-S(a) S(b)$ for $a \in \mathscr{J}_{i j}, b \in \mathscr{J}_{j k}$. 
By (21), $a \times(a \times b)=\left[T\left(a^{\#} \times b\right)+T(a) T(a \times b)\right] 1-T(b) a^{\#}-a^{\#} \times b-T\left(a^{\#}\right) b-T(a \times b) a$ $-T(a) a \times b=T\left(S(a) e_{k} \times b\right) 1-S(a) e_{k} \times b-S(a) b=-S(a) b$ by (33) and (34), so we have

(36) $a \times(a \times b)=-S(a) b$ for $a \in \mathscr{J}_{i j}, b \in \mathscr{J}_{j k}$.

Bilinearize (36) to get

(37) $a \times(b \times c)+b \times(a \times c)=-S(a, b) c$ for $a, b \in \mathscr{J}_{i j}, c \in \mathscr{J}_{j k}$.

Pick $u \in \mathscr{J}_{12}, v \in \mathscr{J}_{31}$ with $S(u) \neq 0, S(v) \neq 0$ and let $\gamma_{12}=-S(u), \gamma_{31}=-S(v)$ and $\gamma_{23}=\left(\gamma_{31} \gamma_{12}\right)^{-1}$. Then $u \times v \in \mathscr{J}_{23}, \quad S(u \times v)=-S(u) S(v)=-\gamma_{12} \gamma_{31}=-\gamma_{23}^{-1}$. Let $w=\gamma_{23} u \times v$, so $S(w)=-\gamma_{23}$. Denote $u, v, w$ by $1_{[12]}, 1_{[31]}, 1_{[23]}$ respectively. By (33) and (36) we have

(38) $1_{[12]}^{\#}=-\gamma_{12} e_{3}, 1_{[23]}^{\#}=-\gamma_{23} e_{1}, 1_{[31]}^{\#}=-\gamma_{31} e_{2}$.

(39) $\gamma_{12} \gamma_{23} \gamma_{31}=1$.

$$
\begin{aligned}
& 1_{[31]} \times 1_{[12]}=\gamma_{31} \gamma_{12} 1_{[23]}, \\
& 1_{[12]} \times 1_{[23]}=\gamma_{12} \gamma_{23} 1_{[31]}, \\
& 1_{[23]} \times 1_{[31]}=\gamma_{23} \gamma_{31} 1_{[12]} .
\end{aligned}
$$

Let $\mathscr{C}=\mathscr{J}_{23} ; \mathscr{C}$ is a $\Phi$ vector space with a distinguished element $1=1_{[23]}$. Define a multiplication in $\mathscr{C}$ by

(41) $a b=\left(1_{[31]} \times a\right) \times\left(1_{[12]} \times b\right)$ for $a, b \in \mathscr{C}=\mathscr{J}_{23}$.

By (40) and (36), $1 b=\left(1_{[31]} \times 1_{[23]}\right) \times\left(1_{[12]} \times b\right)=\gamma_{23} \gamma_{31} 1_{[12]} \times\left(1_{[12]} \times b\right)=\gamma_{23} \gamma_{31} \gamma_{12} b$ $=b$. Similarly $a 1=a$. Bilinearity of the product follows from the definition of $\times$. Define a norm on $\mathscr{C}$ by

(42) $n(a)=-\gamma_{23}^{-1} S(a), a \in \mathscr{C}=\mathscr{J}_{23}$.

Then $n(1)=1$ and $n(a b)=-\gamma_{23}^{-1} S\left(\left(1_{[31]} \times a\right) \times\left(1_{[12]} \times b\right)\right)=\gamma_{23}^{-1} S\left(1_{[31]} \times a\right) S\left(1_{[12]} \times b\right)$ $=\gamma_{23}^{-1} \gamma_{12} \gamma_{31} S(a) S(b)=\gamma_{23}^{-2} S(a) S(b)=n(a) n(b)$ by (35). As $S$ is a nondegenerate quadratic form on $\mathscr{J}_{23}$, so is $n$ on $\mathscr{C}$ and $\mathscr{C}$ is a composition algebra. It has an involution $\bar{a}=t(a) 1-a=n(a, 1) 1-a=-\gamma_{23}^{-1} S(a, 1) 1_{[23]}-a=-\gamma_{23}^{-1} T\left(a \times 1_{[23]}\right) 1_{[23]}-a$.

(43) $\bar{a}=-\gamma_{23}^{-1} T\left(a \times 1_{[23]}\right) 1_{[23]}-a, a \in \mathscr{C}=\mathscr{J}_{23}$.

Define bijective mappings from $\mathscr{C}$ to $\mathscr{J}_{12}$ and $\mathscr{J}_{31}$ :

$$
\begin{array}{rlrl}
\varphi: \mathscr{J}_{23} \rightarrow \mathscr{J}_{31} \text { by } & (a) \varphi & =\gamma_{31} 1_{[12]} \times \bar{a}, \\
\varphi^{-1}: \mathscr{J}_{31} \rightarrow \mathscr{J}_{23} \text { by } & (b) \varphi^{-1} & =\gamma_{23} \overline{1_{[12]} \times b}, \\
\psi: \mathscr{J}_{23} & \rightarrow \mathscr{J}_{12} \text { by } & (a) \psi & =\gamma_{12} 1_{[31]} \times \bar{a}, \\
\psi^{-1}: \mathscr{J}_{12} \rightarrow \mathscr{J}_{23} \text { by } & (c) \psi^{-1} & =\gamma_{23} \overline{1_{[31]} \times c}
\end{array}
$$

where $a \in \mathscr{J}_{23}, b \in \mathscr{J}_{31}, c \in \mathscr{J}_{12}$. Then

$$
a \stackrel{\varphi}{\longrightarrow} \gamma_{31} 1_{[12]} \times \bar{a} \stackrel{\varphi^{-1}}{\longrightarrow} \gamma_{23} \gamma_{31} \overline{1_{[12]} \times\left(1_{[12]} \times \bar{a}\right)}=\gamma_{23} \gamma_{31} \gamma_{12} \overline{\bar{a}}=a
$$

and

$$
b \stackrel{\varphi^{-1}}{\longrightarrow} \gamma_{23} \overline{1_{[12]} \times b} \stackrel{\varphi}{\longrightarrow} \gamma_{23} \gamma_{31} 1_{[12]} \times\left(1_{[12]} \times b\right)=\gamma_{23} \gamma_{31} \gamma_{12} b=b
$$


by (36) and (39). Similarly $(a \psi) \psi^{-1}=a$ and $\left(c \psi^{-1}\right) \psi=c$. Note that the $1_{[i j]}$ 's are mapped onto one another. Denote $a \in \mathscr{C}=\mathscr{J}_{23}$ by $a_{[23]}, a \varphi$ by $a_{[31]}$ and $a \psi$ by $a_{[12]}$. Let

$$
x=\sum_{i=1}^{3} \alpha_{i} e_{i}+\sum_{(123)} a_{i[j k]}
$$

where $\sum_{(123)}$ denotes the sum over cyclic permutations of (123). To compute $x^{\#}$ it suffices to consider the last three terms of $x$ since the others are already known from (33). By (33), $a_{1[23]}^{\#}=S\left(a_{1}\right) e_{1}=-\gamma_{23} n\left(a_{1}\right) e_{1}$. By (10) and (33), $\left(1_{[12]} \times \bar{a}_{2}\right)$ \# $=T\left(1_{[12]}^{\#}, \bar{a}_{2}\right) \bar{a}_{2}+T\left(\bar{a}_{2}^{\#}, 1_{[12]}\right) 1_{[12]}-S\left(\bar{a}_{2}\right) e_{1} \times\left(-\gamma_{12} e_{3}\right)=\gamma_{12} S\left(\bar{a}_{2}\right) e_{2}$, hence

$$
a_{2[31]}^{\#}=\gamma_{31}^{2}\left(1_{[12]} \times \bar{a}_{2}\right)^{\#}=\gamma_{31} \gamma_{23}^{-1} S\left(\bar{a}_{2}\right) e_{2}=-\gamma_{31} n\left(\bar{a}_{2}\right) e_{2}=-\gamma_{31} n\left(a_{2}\right) e_{2} .
$$

Similarly $a_{3[12]}^{\#}=\gamma_{12}^{2}\left(1_{[31]} \times \bar{a}_{3}\right)^{\#}=-\gamma_{12} n\left(a_{3}\right) e_{3}$. By (41),

$$
a_{3[12]} \times a_{2[31]}=\gamma_{12} \gamma_{31}\left(1_{[31]} \times \bar{a}_{3}\right) \times\left(1_{[12]} \times \bar{a}_{2}\right)=\gamma_{23}^{-1}{\overline{\left(a_{2} a_{3}\right.}}_{[23]} .
$$

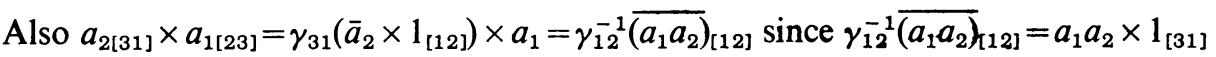
$=\left(\left(a_{1} \times 1_{[31]}\right) \times\left(a_{2} \times 1_{[12]}\right)\right) \times 1_{[31]}$ by $(41)=-\left(\left(a_{1} \times 1_{[31]}\right) \times 1_{[31]}\right) \times\left(a_{2} \times 1_{[12]}\right)-$ $S\left(a_{2} \times 1_{[12]}, 1_{[31]}\right)\left(a_{1} \times 1_{[31]}\right)$ by $(37)=-\gamma_{31} a_{1} \times\left(a_{2} \times 1_{[12]}\right)-S\left(a_{2}, \gamma_{23}^{-1} 1_{[23]}\right)\left(a_{1} \times 1_{[31]}\right)$ by (36), (12) and (13) $=\gamma_{31} a_{1} \times\left(\bar{a}_{2} \times 1_{[12]}\right)$ by (43). Similarly

$$
a_{1[23]} \times a_{3[12]}=\gamma_{12} a_{1} \times\left(1_{[31]} \times \bar{a}_{3}\right)-\gamma_{31}^{-1}{\overline{\left(a_{3} a_{1}\right)}}_{[31]} .
$$

So

$$
\begin{aligned}
x^{\#=} & \left(\alpha_{2} \alpha_{3}-\gamma_{23} n\left(a_{1}\right)\right) e_{1}+\left(\alpha_{3} \alpha_{1}-\gamma_{31} n\left(a_{2}\right)\right) e_{2}+\left(\alpha_{1} \alpha_{2}-\gamma_{12} n\left(a_{3}\right)\right) e_{3} \\
& +\left(\gamma_{23}^{-1} \overline{a_{2} a_{3}}-\alpha_{1} a_{1}\right)_{[23]}+\left(\gamma_{31}^{-1} \overline{a_{3} a_{1}}-\alpha_{2} a_{2}\right)_{[31]}+\left(\gamma_{12}^{-1} \overline{a_{1} a_{2}}-\alpha_{3} a_{3}\right)_{[22]} .
\end{aligned}
$$

Let $\gamma_{1}$ be an arbitrary nonzero element of $\Phi, \gamma_{2}=\gamma_{1} \gamma_{12}^{-1}, \gamma_{3}=\gamma_{1} \gamma_{31}$, so $\gamma_{12}=\gamma_{2}^{-1} \gamma_{1}$, $\gamma_{31}=\gamma_{1}^{-1} \gamma_{3}$ and $\gamma_{23}=\gamma_{3}^{-1} \gamma_{2}$. Then if

$$
X=\left(\begin{array}{ccc}
\alpha_{1} & a_{3} & \gamma_{1}^{-1} \gamma_{3} \bar{a}_{2} \\
\gamma_{2}^{-1} \gamma_{1} \bar{a}_{3} & \alpha_{2} & a_{1} \\
a_{2} & \gamma_{3}^{-1} \gamma_{2} \bar{a}_{1} & \alpha_{3}
\end{array}\right) \in \mathscr{H}\left(\mathscr{C}_{3}, \mathscr{J}_{\gamma}\right),
$$

$X$ has the same \# as the above element $x \in \mathscr{J}(N, \#, 1)$. The two algebras have the same underlying spaces and trace form $T$ (by (33)), one needs only $T$ on the Peirce spaces and $T\left(a_{[i j]}, b_{[i j]}\right)=T\left(a_{[i j]}\right) T\left(b_{[i j]}\right)-T\left(a_{[i j]} \times b_{[i j]}\right)=\gamma_{i j} t(a, b)$ (by (44)) and therefore the same $U$. Thus they are isomorphic and this completes the proof of Theorem 1. (Note that (32) and (3) imply that the norms are the same.)

3. Springer's Theorem. In [2] Faulkner proves the following theorem.

THEOREM 2 (ALBERT-JACOBSON). Let $\mathscr{J}$ be a reduced central simple exceptional quadratic Jordan algebra. If $\mathscr{J} \cong \mathscr{H}\left(\mathscr{C}_{3}, J_{\gamma}\right)$ and $\mathscr{J} \cong \mathscr{H}\left(\mathscr{C}_{3}^{\prime}, J_{\gamma^{\prime}}\right)$ then $\mathscr{C} \cong \mathscr{C}^{\prime}$.

We wish to give next a simple proof of Springer's Theorem. 
THEOREM 3 (SPRINGER [9]). Let $\mathscr{J}$ and $\mathscr{J}^{\prime}$ be reduced central simple exceptional quadratic Jordan algebras, $\mathscr{J} \cong \mathscr{H}\left(\mathscr{C}_{3}, J_{\gamma}\right), \mathscr{J}^{\prime} \cong \mathscr{H}\left(\mathscr{C}_{3}^{\prime}, J_{\gamma^{\prime}}\right)$ then $\mathscr{J} \cong \mathscr{J}^{\prime}$ if and only if $\mathscr{C} \cong \mathscr{C}^{\prime}$ and $\mathscr{J}$ and $\mathscr{J}^{\prime}$ have equivalent quadratic forms $S$.

Springer [9] uses $Q(x)=\frac{1}{2} T\left(x^{2}\right)$ rather than $S(x)$, however, apart from considerations of characteristic, $S(x)$ seems more natural in view of Lemma 2 and of [6]. His proof makes use of spin groups and can also be found in Chapter 9 of [3]. McCrimmon [7] has given shorter proofs of Theorems 2 and 3 when the characteristic of $\Phi \neq 2$.

Necessity follows from the Albert-Jacobson Theorem. We have $\mathscr{J} \cong \mathscr{H}\left(\mathscr{C}_{3}, J_{\gamma}\right)$, $\mathscr{J}^{\prime} \cong \mathscr{H}\left(\mathscr{C}_{3}, J_{\gamma^{\prime}}\right)$. If $\mathscr{C}$ is split then $n(\mathscr{C})=\Phi$ and it follows from the proof of Theorem 1 that $\gamma_{12}$ and $\gamma_{31}$ can be picked $=1$, so that $\gamma_{23}=1$ and $\mathscr{J} \cong \mathscr{H}\left(\mathscr{C}_{3}\right)$, similarly for $\mathscr{J}^{\prime}$. So $\mathscr{C}$ split implies that all algebras $\mathscr{H}\left(\mathscr{C}_{3}, J_{\gamma}\right)$ are isomorphic (to $\left.\mathscr{H}\left(\mathscr{C}_{3}\right)\right)$. We may therefore assume that $\mathscr{C}$ is a division octonion algebra. We will need the Witt-Arf Theorem, e.g. [9, p. 12]. Let $Q$ be a nondegenerate quadratic form on a $\Phi$ vector space $V, R=\{x \in V \mid Q(x, V)=0\}$ the radical of $Q$. An isometry $s$ of a subspace $W$ into $V$ is called admissible if $s$ can be extended to an isometry $s^{\prime}$ of $W+R$ in $V$ such that $s^{\prime}$ is the identity on $R$.

TheOREM (WITT-Arf). Any admissible isometry of a subspace $W$ into $V$ can be extended to an orthogonal transformation of $V$.

Let $\mathscr{J}=\mathscr{H}\left(\mathscr{C}_{3}, J_{\gamma}\right)$. Let $x=\sum_{i=1}^{3} \alpha_{i} e_{i}+\sum_{(123)} a_{i[j k]}, \quad y=\sum_{i=1}^{3} \beta_{i} e_{i}+\sum_{(123)} b_{i[j k]}$, it follows easily from (44) that $S(x, y)=\sum_{(123)}\left[\left(\alpha_{i}+\alpha_{j}\right) \beta_{k}-\gamma_{j}^{-1} \gamma_{i} t\left(a_{k}, b_{k}\right)\right]$. If $S(x, \mathscr{J})$ $=0$, the nondegeneracy of $t$ implies $a_{1}=a_{2}=a_{3}=0$ and $\alpha_{1}+\alpha_{2}=\alpha_{2}+\alpha_{3}=\alpha_{3}+\alpha_{1}=0$. Hence the radical of $S=\{0\}$ if the characteristic of $\Phi \neq 2, \Phi 1$ if the characteristic of $\Phi=2$. We wish to show that any primitive idempotent $e$ of $\mathscr{J}$ can be embedded in a system of mutually orthogonal idempotents. By the argument on p. 97, we may assume that the characteristic of $\Phi$ is 2 and we must show that $T\left(\mathscr{J}_{0}\right) \neq 0$. Since $f$ does not belong to the radical of $S, S(x, f) \neq 0$ for some $x \in \mathscr{J}$. But $S(x, f)$ $=T(x \times f)=T(x) T(f)-T(x, f)=2 T(x)-T(x, f)=T(x, f)$. By (25) we may assume $x \in \mathscr{J}_{0}$. Therefore $T(x)=T(x, f) \neq 0$ and $T\left(\mathscr{J}_{0}\right) \neq 0$.

Let $e_{1}$ be a primitive idempotent of $\mathscr{J}$. Pick $e_{2} \perp e_{3}$ primitive idempotents of $\mathscr{J}_{0}\left(e_{1}\right) ; \mathscr{J}_{0}\left(e_{1}\right)=\Phi e_{2} \oplus \Phi e_{3} \oplus \mathscr{J}_{23}, S\left(\alpha_{2} e_{2}+\alpha_{3} e_{3}+a_{1[23]}\right)=\alpha_{2} \alpha_{3}-\gamma_{3}^{-1} \gamma_{2} n\left(a_{1}\right)$. Define the norm class of $e_{1}$ to be $\kappa\left(e_{1}\right)=-\gamma_{3}^{-1} \gamma_{2} n(\dot{\mathscr{C}})$, where $\dot{\mathscr{C}}=\mathscr{C}-\{0\}$. By the Witt-Arf Theorem, $\kappa\left(e_{1}\right)$ depends only on the restriction of $S$ to $\mathscr{J}_{0}\left(e_{1}\right)$.

LeMmA 3. Let $\mathscr{J} \cong \mathscr{H}\left(\mathscr{C}_{3}, J_{\gamma}\right), \mathscr{J}^{\prime} \cong \mathscr{H}\left(\mathscr{C}_{3}, J_{\gamma^{\prime}}\right)$ with equivalent forms $S$, then any two primitive idempotents $e \in \mathscr{J}$ and $e^{\prime} \in \mathscr{J}^{\prime}$ with $\kappa(e)=\kappa\left(e^{\prime}\right)$ may be mapped into each other by an isomorphism of $\mathscr{J}^{\prime}$ onto $\mathscr{J}$.

Proof. We may assume that $e=e_{1}, e^{\prime}=e_{1}^{\prime}$ where $e_{1}, e_{2}, e_{3}$ and $e_{1}^{\prime}, e_{2}^{\prime}, e_{3}^{\prime}$ are the idempotents in the above coordinatizations of $\mathscr{J}$ and $\mathscr{J}^{\prime}$. Since $\kappa(e)=\kappa\left(e^{\prime}\right)$ there 
is an isometry between $\mathscr{J}_{23}^{\prime}$ and $\mathscr{J}_{23}$ which can then be extended to an isometry of $\Phi e_{1}^{\prime} \oplus \Phi e_{2}^{\prime} \oplus \Phi e_{3}^{\prime} \oplus \mathscr{J}_{23}^{\prime}=W^{\prime}$ to $\Phi e_{1} \oplus \Phi e_{2} \oplus \Phi e_{3} \oplus \mathscr{J}_{23}=W$. So the Witt-Arf Theorem implies that the above isometry can be extended to yield an isometry of $W^{\prime \perp}=\mathscr{J}_{12}^{\prime}+\mathscr{J}_{31}^{\prime}$ to $W^{\perp}=\mathscr{J}_{12} \oplus \mathscr{J}_{31}$. Thus there exists an $x \in W^{\perp}$ with $S(x)$ $=-\gamma_{2}^{\prime-1} \gamma_{1}^{\prime}=S\left(1_{[12]}^{\prime}\right), 1_{[12]}^{\prime} \in J_{12}^{\prime} ; S(x)=T\left(x^{\#}\right)$, so $x^{\#} \neq 0, x^{\#} \in \mathscr{J}_{0}\left(e_{1}\right), x^{\# \#}=0$ therefore $g_{3}=S(x)^{-1} x^{\#}$ is a primitive idempotent in $\mathscr{J}_{0}\left(e_{1}\right)$. Let $g_{1}=e_{1}, g_{2}=1-g_{1}-g_{3}$; by (28) and (5) $x \in \mathscr{J}_{1 / 2}\left(g_{1}\right)$ implies $\left(g_{2}+g_{3}\right) \times x=-x$. But by (14), (26) and (32), $g_{3} \times x$ $=S(x)^{-1} x^{\#} \times x=S(x)^{-1}\left(\left[T\left(x^{\#}\right) T(x)-N(x)\right] 1-T\left(x^{\#}\right) x-T(x) x^{\#}\right)=-S(x)^{-1} T\left(x^{\#}\right) x=$ $-x$. Therefore $g_{2} \times x=0$ and since $T(x)=0,(28)$ implies $x \in \mathscr{J}_{1 / 2}\left(g_{2}\right) ; x \in \mathscr{J}_{1 / 2}\left(g_{1}\right)$ $\cap \mathscr{J}_{1 / 2}\left(g_{2}\right)=\mathscr{J}_{12}$ (re the $g_{i}^{\prime}$ s), $S(x)=-\gamma_{2}^{\prime-1} \gamma_{1}^{\prime}$. We still have $\kappa\left(g_{1}\right)=\kappa\left(e_{1}\right)=\kappa\left(e_{1}^{\prime}\right)$, so there is a $y \in \mathscr{J}_{23}$ with $S(y)=-\gamma_{3}^{\prime-1} \gamma_{2}^{\prime}$. This yields a coordinatization of $\mathscr{J}$ re $e_{1}$ $=g_{1}, g_{2}, g_{3}$ with the same $\gamma_{i}^{\prime \prime}$ s as the coordinatization of $\mathscr{J}^{\prime}$.

COROllary. Let $\mathscr{J}$ be as above, then two primitive idempotents $e$ and $e^{\prime}$ are in the same orbit under the automorphism group of $\mathscr{J}$ if and only if $\kappa(e)=\kappa\left(e^{\prime}\right)$.

The following lemma will complete the proof of Theorem 3.

LEMMA 4. If $\mathscr{J} \cong \mathscr{H}\left(\mathscr{C}_{3}, J_{\gamma}\right), \mathscr{J}^{\prime} \cong \mathscr{H}\left(\mathscr{C}_{3}, J_{\gamma^{\prime}}\right)$ have equivalent forms $S$ then there exist primitive idempotents $e \in \mathscr{J}, e^{\prime} \in \mathscr{J}^{\prime}$ with $\kappa(e)=\kappa\left(e^{\prime}\right)$.

Proof. Assume $e^{\prime}=e_{1}^{\prime}$, then $\kappa\left(e^{\prime}\right)=-\gamma_{3}^{\prime}-1 \gamma_{2}^{\prime} n(\dot{\mathscr{C}})$. The Witt-Arf Theorem allows us to extend the isometry between $\Phi e_{1}^{\prime} \oplus \Phi e_{2}^{\prime} \oplus \Phi e_{3}^{\prime}$ and $\Phi e_{1} \oplus \Phi e_{2} \oplus \Phi e_{3}$ by an isometry between $\mathscr{J}_{12}^{\prime} \oplus \mathscr{J}_{23}^{\prime} \oplus \mathscr{J}_{31}^{\prime}$ and $\mathscr{J}_{12} \oplus \mathscr{J}_{23} \oplus \mathscr{J}_{31}$. So there is an $x \in \mathscr{J}_{12} \oplus \mathscr{J}_{23} \oplus \mathscr{J}_{31}$ with $S(x)=-\gamma_{3}^{-1} \gamma_{2}$. If $x \in \mathscr{J}_{1 / 2}\left(e_{i}\right)$ for some $i=1,2$ or 3 then argue as in the proof of Lemma 3 to get a primitive idempotent $e$ with $\kappa(e)=S(x) n(\dot{\mathscr{C}})$. If not then $x=a_{1[23]}+a_{2[31]}+a_{3[12]}$ with $n\left(a_{1}\right) n\left(a_{2}\right) n\left(a_{3}\right) \neq 0$,

$$
S(x)=-\left[\gamma_{3}^{-1} \gamma_{2} n\left(a_{1}\right)+\gamma_{1}^{-1} \gamma_{3} n\left(a_{2}\right)+\gamma_{2}^{-1} \gamma_{1} n\left(a_{3}\right)\right],
$$

$N(x)=t\left(a_{1} a_{2} a_{3}\right)$. We proceed to show that we may assume $N(x)=0$. If this is not the case, recoordinatizing if necessary, we may assume $a_{1}=a_{2}=1$. Pick $b \in \dot{\mathscr{C}}$ with $t\left(b a_{3}\right)=t\left(\bar{b}, a_{3}\right)=0$. Consider $y=b_{[23]}+\bar{b}_{[31]}+b a_{3[12]}, \quad S(y)=n(b) S(x)$ and $N(y)$ $=t\left(b \bar{b}\left(b a_{3}\right)\right)=n(b) t\left(b a_{3}\right)=0$. Thus we may assume that we have an $x \in \mathscr{J}_{12} \oplus \mathscr{J}_{23}$ $\oplus \mathscr{J}_{31}$ with $S(x) \in \kappa\left(e^{\prime}\right), N(x)=0$; so $x^{\# \#}=0$ and $g=S(x)^{-1} x^{\#}$ is a primitive idempotent. By (14) $g \times x=-x$ and since $T(x)=0$, (29) implies $x \in \mathscr{J}_{0}(g)$. If $T\left(e_{1}, g\right)=1$ then the coefficient of $e_{1}$ in $x^{\#}$ is $S(x)$ and letting $y=a_{1[23]}, y^{\#}=S(y) e_{1}=S(x) e_{1}$ and $\kappa\left(e^{\prime}\right)=\kappa\left(e_{1}\right)$. If not, consider $e_{1} U_{f}$ where $f=1-g ; e_{1} U_{f} \in \mathscr{J}_{0}(g),\left(e_{1} U_{f}\right)^{\#}=e_{1}^{\#} U_{f \#}=0$, $T\left(e_{1} U_{f}\right)=T\left(e_{1} U_{f}, 1\right)=T\left(e_{1}, f\right)=T\left(e_{1}, 1-g\right)=1-T\left(e_{1}, g\right) \neq 0$. Therefore $g_{2}=$ $T\left(e_{1} U_{f}\right)^{-1} e_{1} U_{f}$ is a primitive idempotent in $\mathscr{J}_{0}(g), T\left(e_{1} U_{f}, x\right)=T\left(e_{1}, x U_{f}\right)=T\left(e_{1}, x\right)$ $=0$. Now $x \in \mathscr{J}_{0}(g), T(x)=0$ and $T\left(g_{2}, x\right)=0$ imply $x \in\left(\Phi g_{2}+\Phi g_{3}\right)^{\perp} \cap \mathscr{J}_{0}(g)$ where $g_{3}=f-g_{2}$ and we have $\kappa(g)=\kappa\left(e^{\prime}\right)$. This completes the proof of the lemma. 


\section{REFERENCES}

1. F. van der Blij and T. A. Springer, The arithmetics of octaves and of the group $G_{2}$, Nederl. Akad. Wetensch. Proc. Ser. A 62=Indag. Math. 21 (1959), 406-418. MR 27 \#2533.

2. J. Faulkner, Octonion planes defined by quadratic Jordan algebras, Mem. Amer. Math. Soc. No. 104 (1970).

3. N. Jacobson, Structure and representations of Jordan algebras, Amer. Math. Soc. Colloq. Publ., vol. 39, Amer. Math. Soc., Providence, R. I., 1968. MR 40 \#4330.

4. - - Structure theory of quadratic Jordan algebras, Lecture Notes, Tata Institute, Bombay, 1970.

5. N. Jacobson and $\mathrm{K}$. McCrimmon, Quadratic Jordan algebras of quadratic forms with base points, Indian $\mathrm{J}$. (to appear).

6. K. McCrimmon, The Freudenthal-Springer-Tits constructions of exceptional Jordan algebras, Trans. Amer. Math. Soc. 139 (1969), 495-510. MR 39 \#276.

7. —-, A note on reduced Jordan algebras, Proc. Amer. Math. Soc. 19 (1968), 964-970. MR 37 \#2826.

8. T. A. Springer, On a class of Jordan algebras, Nederl. Akad. Wetensch. Proc. Ser. A 62 =Indag. Math. 21 (1959), 254-264. MR 22 \#1607.

9. — The classification of reduced exceptional simple Jordan algebras, Nederl. Akad. Wetensch. Proc. Ser. A 63=Indag. Math. 22 (1960), 414-422. MR 26 \#5035.

10. T. Tamagawa, Mimeographed notes on quadratic forms, Yale University, New Haven, Conn., 1969.

Department of Mathematics, Yale University, New Haven, Connecticut 06520

Current address: Department of Mathematics, Carleton University, Ottawa 1, Ontario, Canada 